\title{
Combining mechanism and drift in community ecology: a novel statistical mechanics approach.
}

\author{
J. Bertram • R. C. Dewar
}

Received: date / Accepted: date

\begin{abstract}
A key challenge for models of community ecology is to combine deterministic mechanism and stochastic drift in a systematic, transparent and tractable manner. Another challenge is to explain and unify different ecological patterns, hitherto modelled in isolation, within a single modelling framework. Here we show that statistical mechanics provides an effective way to meet both challenges. We apply the statistical principle of maximum entropy (MaxEnt) to a simple resource-based, non-neutral model of a plant community. In contrast to previous ecological applications of MaxEnt, our use of MaxEnt emphasises its theoretical basis in the combinatorics of sampling frequencies, an approach that clarifies its ecological interpretation. In this approach mechanism and drift are identified, respectively, with ecological resource constraints and entropy maximization. We obtain realistic predictions for species abundance distributions as well as contrasting stability-diversity relationships at community and population levels. The model also predicts critical behaviour that may provide a basis for understanding desertification and other ecological tipping points. Our results complement and extend previous ecological applications of MaxEnt to new areas of community ecology, and further illustrate MaxEnt as a powerful yet simple modelling tool for combining mechanism and drift in a way that unifies disparate ecological patterns.
\end{abstract}

Keywords Community ecology $\cdot$ statistical mechanics · ecological drift · maximum entropy

J. Bertram · R. C. Dewar

Research School of Biology, Australian National University, Canberra, ACT 0200, Australia

E-mail: jason.bertram@anu.edu.au

R. C. Dewar

E-mail: roderick.dewar@anu.edu.au

\section{Introduction}

Many ecological processes are so complicated and unpredictable that modelling them in detail is difficult or impossible (typical examples are death, reproduction, dispersal, disturbances and the influx of vital resources). For these processes it is far simpler to adopt a stochastic approach, using probabilities to represent their unpredictable nature (Bartlett, 1960). However, it is not always desirable to model ecosystem behaviour stochastically: deterministic biological mechanisms in the form of interactions between functionally distinct groups of organisms and their environment are thought to underpin important aspects of ecosystem behaviour (Vellend, 2010). When mechanisms of this kind are known or assumed to be important, it is natural to attempt to model them in a relatively detailed, deterministic manner. As a result, a recurring theme in theoretical ecology is the trade-off between modelling biological details explicitly (collectively labelled "mechanism") in order to retain model realism versus modelling those details stochastically (collectively labelled "drift") in order to avoid excessive model complexity. This forms part of a broader, long running debate about the respective roles of biological mechanism and drift in ecology which dates back to Darwin's famous "entangled bank" (Maurer, 1999; McGill et al, 2005; McGill, 2006; Adler et al, 2007; McGill and Nekola, 2010; Vellend, 2010; Rosindell et al, 2012; Clark, 2012).

Without going into the specifics of this debate, it is clear that to understand how the interplay between biological mechanism and drift governs the functioning of ecosystems, ecological models need to combine mechanistic and stochastic modelling approaches in a systematic, transparent and tractable manner. Yet, existing "combined" models (e.g. stochastic resource competi- 
tion models (Tilman, 2004) or "almost neutral" models (Zhou and Zhang, 2008)) tend to be significantly more complicated than their purely mechanistic (e.g. Lotka-Volterra) or purely stochastic (e.g. neutral theory (Hubbell, 2001)) counterparts. Specifically, these combined models typically involve assigning probabilities (or probability distributions) to particular variables or processes in a largely ad hoc manner, and are generally challenging to implement (e.g. requiring many repeated numerical calculations with different sample values of the stochastic variables).

In this paper, we introduce a novel approach to the problem of modelling the interplay between mechanism and drift in ecological communities. This approach is based on statistical mechanics, a set of theoretical tools originally developed in physics. In statistical mechanics, the behaviour of a system at a given scale is predicted as the emergent statistical outcome of the behaviour of its constituents at smaller scales, without representing smaller-scale processes in all their detail (Landau and Lifschitz, 1958). This distinctive "top down" perspective can be contrasted with the "bottom-up" representation of community behaviour in many ecological models explicitly in terms of organism-level processes (e.g. stochastic birth, death and dispersal in neutral theory). The practical advantage of statistical mechanics, in physics or ecology, rests on the assumption that only a small, restricted subset of the underlying dynamics needs to be represented explicitly in order to successfully predict system-level behaviour. It is this small subset of the dynamics that we label "mechanism". The countless other details (e.g. of organismal and environmental dynamics) are effectively treated stochastically as drift. As a result, statistical mechanics provides a natural framework for combining mechanism and drift, by representing a few key mechanistic details explicitly while representing the influence of other (potentially very complicated) processes implicitly as drift.

The idea of adapting the methods of statistical physics to ecology is far from new - Lotka proposed this almost a century ago (Lotka, 1925, p. 39) - and a small but growing number of ecological models have (or proclaim to have) derived inspiration from statistical mechanics (e.g. Kerner, 1957; Lurié and Wagensberg, 1983; Alexeyev and Levich, 1997; Volkov et al, 2004; Shipley et al, 2006; Pueyo et al, 2007; Harte et al, 2008; Dewar and Porté, 2008; Banavar et al, 2010). Yet, in spite of their apparent similarities, these models differ considerably in both approach and area of application, and Lotka's proposal remains largely unexplored. More specifically, several previous models have based their rationale for using statistical mechanics on physical analogy (e.g. the dynamical invariants approach of
Kerner (1957)) or appeal to the more general framework of information theory (e.g. Dewar and Porté (2008)). However, the ecological interpretation of these rather abstract approaches is not particularly transparent (for more discussion, see section 4).

The aim of this study is two-fold. First, we seek to introduce a statistical mechanics approach to ecological modelling which is grounded solely in ecological concepts and assumptions, without appeal to physical analogy or information theory. In common with several previous models we also use the principle of maximum entropy (MaxEnt); however, we stress the theoretical basis of MaxEnt in the combinatorics of sample frequencies. This sample frequency basis for MaxEnt combines mechanism and drift in a transparent manner.

Secondly, we aim to illustrate our approach in a specific ecological setting, by applying MaxEnt to an idealised, trait-based model of a plant community. The model developed here is adapted, with significant modifications, from the general approach of Volkov et al (2004), Dewar and Porté (2008) and Bertram and Dewar (2013). In the model, the land area occupied by a plant community is uniformly divided into a large number of grid cells, each grid cell being in a state characterised by one out of a finite number of possible cover types. It is assumed that different cover types have distinct mean-annual requirements of one critical resource (e.g. water or nitrogen) and that the corresponding total community resource requirement balances total community resource availability when averaged over many years. Together, these two assumptions constitute the mechanistic element of the model. Given these biological assumptions, we then use MaxEnt to calculate the joint probability that the different cover types have given abundances, which effectively amounts to assuming that cover type abundances vary randomly from year to year within the constraints imposed by mean annual resource balance. This constrained drift in cover type abundances is reflected in the predicted joint probability distribution for cover type abundances, which exhibits significant variability about its mean.

To evaluate the predictions of our model, we compare them with well-known ecological patterns, specifically species abundance distributions (SADs) and stability-diversity relationships (i.e. the changes in abundance variances with increasing diversity). We also show that our model exhibits a "phase transition" corresponding to a sudden onset of desertification i.e. a sudden increase in the fraction of non-vegetated land area as resource availability is reduced - similar behaviour has been documented in arid ecosystems (Scheffer et al, 2001; Kéfi et al, 2007). Each of these different aspects of plant ecosystem behaviour (species 
abundance patterns, stability-diversity relationships, desertification) is important in its own right, and so it interesting to see how our model performs in each case. However, more importantly, these different aspects have so far been studied largely in isolation from one another: a huge body of ecological theory (including MaxEntbased models) focuses almost exclusively on abundance patterns like SADs (McGill et al, 2007; McGill, 2010); stability-diversity relationships have been broadly interpreted in terms of scaling laws between abundance means and variances (e.g. the portfolio effect); and discussion of ecological "catastrophic shifts" in the context of desertification has been largely descriptive (Scheffer et al, 2001; Kéfi et al, 2007). The novel contribution of this study is that for the first time these different areas of community ecology are brought together within a single MaxEnt-based model, and with remarkable success given the simplicity of the model. These results extend and complement previous studies by Dewar and Porté (2008) and Bertram and Dewar (2013), in which closely-related MaxEnt-based models were used to predict other ecological patterns, including contrasting diversity-productivity relationships at different spatial scales, energetic equivalence and power law self-thinning behaviour in resource-rich communities, and tree-grass co-existence patterns as a function of water availability in tropical savannas.

The paper is structured as follows. In section 2 we discuss the basic physiological assumptions of our model as well as the joint probability distribution for cover type abundances. The sample frequency interpretation of MaxEnt is introduced with an emphasis on how this relates to the ecological interpretation of the cover type probability distribution, as well as the division between mechanism and drift. In section 3 we use the model developed in section 2 to predict SADs, stability-diversity relationships and critical behaviour during a transition from a predominantly vegetated to a predominantly non-vegetated state. We summarise our conclusions in section 4 . Key analytical results are given in the main text; full mathematical details and proofs are confined to Appendices A-E.

\section{Model description}

2.1 Grid cells, cover types and resource consumption

An idealised plant community occupying a fixed area (of order $1 \mathrm{~km}^{2}$ ) is uniformly divided into a large number $N$ of grid cells (of order $1 \mathrm{~m}^{2}$ ). Each grid cell is occupied by either bare ground, or one out of $C$ possible plant cover types. Cover type abundances at a given time are denoted $n_{i}$, where $i=0, \ldots, C$ (i.e. $n_{i}$ is the number of grid cells of cover type $i$ ). These abundances satisfy

$n_{0}+\ldots+n_{C}=N$.

We assume for simplicity that the plants in the community depend primarily on one critical resource for survival and reproduction (e.g. water or nitrogen). The approach can easily be generalised to multiple resources. Each year different amounts $R(\mathrm{~kg} / \mathrm{yr})$ of this resource become available to the community. Averaging these amounts over many years defines the mean annual community resource availability $\bar{R}$ (henceforth an overline denotes the mean annual average).

We assume that each cover type $i$ requires a distinct, fixed amount $r_{i}(\mathrm{~kg} / \mathrm{yr})$ of the resource per grid cell per year. For example, if the critical resource is water then $r_{i}$ is the annual evapotranspiration (evaporation plus transpiration) of a grid cell of cover type $i$ (this reduces to evaporation alone in the case of grid cells occupied by bare ground). For convenience, cover types are labelled in the order of their resource use $r_{0}<r_{1}<\ldots<r_{C}$, and the scale of resource use is shifted so that $r_{0}=0$ (i.e. subtract $r_{0}$ from all resource use values so that they represent values relative to cover type $i=0$ ). Note that this fixed resource use assumption implies that grid cell resource requirements are independent of each other i.e. a grid cell with cover type $i$ has resource requirement $r_{i}$ independently of the cover types occupying other grid cells in the community.

In this generic model, different cover types represent functional differences in the consumption of a critical resource. Thus, any grid cell that uses an amount $r_{i}$ of the critical resource in a given year is regarded as belonging to cover type $i$ in that year, regardless of its biotic composition. Cover type differences could reflect broad functional categories such as grasses versus trees or $C_{3}$ versus $C_{4}$ photosynthesis, different species, different phenotypes or developmental stages within a species, or some combination of these. Thus, our generic approach differs from the more traditional approach of viewing organisms as the elements of a community and distinguishing them according to taxonomically-defined species. In certain circumstances these approaches coincide. For example, if all plants have approximately the same size and each species has a distinct, fixed resource requirement, then we can identify grid cells with individuals and cover types with species. However, since the sizes of individual plants can differ considerably, it is mathematically simpler to choose uniform grid cells as the community elements rather than individual plants (see Bertram and Dewar (2013) for a similar non-uniform grid cell model). 
Furthermore, it is natural to distinguish community elements functionally from the outset in order to capture the influence of functional differences on community structure. Differences between taxonomicallydefined species need not be the most important ones from a functional point of view. Indeed, the relative lack of diversity in the average traits of different species compared with the trait diversity thought to be required to sustain observed species richness is the familiar "biodiversity paradox" (Clark et al, 2007). In forest communities, intra-specific variation may far exceed interspecific variation in growth-rates (Clark, 2010).

From the above assumptions, it follows that the annual community resource consumption associated with the abundance vector $\mathbf{n}=\left(n_{0}, \ldots, n_{C}\right)$ is given by

$r(\mathbf{n})=n_{1} r_{1}+\ldots+n_{C} r_{C}$.

(Recall that $r_{0}=0$ ). This resource consumption does not necessarily balance the amount of that resource made available to the community in a given year, denoted $R$. Some of the resources made available may not be consumed $(r<R)$ or, if the community has a reserve of resources, more resources may be consumed than are made available $(r>R)$. However, we assume mean annual resource balance

$\bar{r}=\bar{R}$.

Equation (3) states that the mean annual rate of resource use by the community equals the mean annual rate of resource supply to the community. The timescale on which this balance may be expected to occur will depend on the nature of the resource; for water, it may be of the order of $5-10$ years.

Finally, we assume that the $i=0$ cover type, which uses the least resources, corresponds to bare ground. This assumption is reasonable because we expect that fewer resources will be consumed in the absence of plants using resources for survival and reproduction. Returning to the water example, bare ground evaporation will generally be smaller than evapotranspiration from an equivalent area of vegetated ground because vegetation accesses deeper soil moisture to sustain transpiration (Bertram and Dewar, 2013).

The resource use values $r_{0}, \ldots, r_{C}$ must be specified to make quantitative predictions. To explore some illustrative cases, we will consider the case where resource consumption takes the form of a power law

$r_{i}=r_{C}\left(\frac{i}{C}\right)^{\gamma}$

and consider different choices for $\gamma$. This power law represents three qualitatively distinct regimes: uniformly spaced $r_{i}(\gamma=0) ; r_{i}$ bunched near the greatest resource use $r_{C}(\gamma<1)$; and $r_{i}$ bunched near the bare ground resource use $r_{0}=0(\gamma>1)$. Equation (4) is a convenient parametrisation for exploring the predictions of our model and should not be interpreted as claiming that cover type resource use follows a neat power law in actual communities (although relationships similar to (4) have previously been derived from allometric scaling arguments (Dewar and Porté, 2008)). The cover types present in actual communities could possibly exhibit a more complicated resource use structure e.g. with irregular spacing between the $r_{i}$, and in general the quantitative predictions of our model will depend to some extent on the details of that structure. However, the analytical results presented in section 3 are valid for arbitrary $r_{i}$. Moreover, the numerical results presented in section 3 are largely insensitive the detailed structure of the $r_{i}$; in contrast, they do depend on major qualitative differences in "bunching" as expressed by (4) with different $\gamma$.

\subsection{Predicting abundance probabilities with MaxEnt}

The key quantity to be predicted from our model is the joint probability distribution of cover type abundances; specifically, the joint abundance sample frequency distribution (SFD) defined as the proportion of time that the community has abundance vector $\mathbf{n}$. To do so, we apply a sample frequency formulation of the principle of Maximum Entropy (MaxEnt), as follows.

Suppose that the grid cell abundance vector in our plant community is measured each year over a large number $(M)$ of years yielding an abundance sequence $\mathbf{n}_{1}, \mathbf{n}_{2}, \ldots, \mathbf{n}_{M}$. Then the corresponding sample frequency distribution is given by $p(\mathbf{n})=m(\mathbf{n}) / M$ where $m(\mathbf{n})$ is the number of years that $\mathbf{n}$ appears in this sequence. $M$ is assumed to be large to ensure that the relative sample frequencies $p(\mathbf{n})$ accurately reflect the long-run average behaviour of the community.

Reordering the abundance vectors in an abundance sequence does not change the resulting SFD, and so a given $p(\mathbf{n})$ is realised by many different abundance sequences. Futhermore, some SFDs can be realised by a greater number of abundance sequences than others. In the limit where $M$ is large, it can easily be shown (Jaynes and Bretthorst, 2003) that the Shannon entropy

$$
H=-\sum_{n_{0}+\ldots+n_{C}=N} p(\mathbf{n}) \ln p(\mathbf{n})
$$

is a logarithmic measure of $W$, the number of sample sequences that realise a given distribution $p(\mathbf{n})$; specifically, $W \rightarrow \exp (M H)$ as $M \rightarrow \infty$. Consequently, the 
SFD that can be realised by the greatest number of sequences is the one that maximises $H$ with respect to $p(\mathbf{n})$, denoted $p_{\mathrm{ME}}(\mathbf{n})$ (ME=Maximum Entropy). Moreover, since $M$ is large, $p_{\mathrm{ME}}(\mathbf{n})$ is realised by the overwhelming majority of possible sample sequences.

The preceding combinatorial argument also applies if constraints are placed on $p(\mathbf{n})$, thereby restricting the set of abundance sequences. Maximising $H$ subject to constraints on $p(\mathbf{n})$ selects the SFD realised by the overwhelming majority of sequences that are consistent with those constraints.

The ecological significance of maximising $H$ subject to constraints on $p(\mathbf{n})$ is as follows. If the abundances in our plant community are entirely subject to the whims of drift - i.e. if measured abundance sequences appear to be selected at random - then the corresponding SFD will almost certainly be equal to the unconstrained SFD $p_{\mathrm{ME}}(\mathbf{n})$ because it represents the overwhelming majority of possible sample sequences. Constraints on $p(\mathbf{n})$ represent key ecological factors that restrict the domain of abundance sequences within which drift can operate. Thus, by maximising $H$ subject to constraints on $p(\mathbf{n})$, we are effectively assuming that abundances are governed entirely by drift within the boundaries imposed by the constraints. The joint abundance probability SFD of the community is thereby decomposed into two contributions: one due to mechanism (constraints) and one due to drift (maximum $H$ ).

Notice that in this conception of drift, abundances are random and independent from year to year (within the imposed constraints). This differs from the "random walk" conception of drift familiar in stochastic process models, in which abundances randomly change in increments so that future abundances depend on their value in previous years.

\subsection{The role of mean annual resource balance}

The unconstrained, pure drift case represented by $p_{\mathrm{ME}}(\mathbf{n})$ does not reflect realistic community behaviour. Indeed, $p_{\mathrm{ME}}(\mathbf{n})$ is a uniform distribution over $\mathbf{n}$ (analogous to the expected $50: 50$ distribution of heads and tails in a long sequence of unbiased coin tosses); in actual communities some cover types are typically more abundant than others on average and thus the joint abundance SFD will not be uniform.

Within the setting of our plant community model (section 2.1) this result is not surprising because grid cell abundances cannot be governed by drift alone: grid cells have different resource requirements and mean annual resource balance must be satisfied. From equation
(3), this means that $p(\mathbf{n})$ must satisfy

$$
\sum_{n_{0}+\ldots+n_{C}=N} p(\mathbf{n}) r(\mathbf{n})=\bar{R} .
$$

(By definition, the mean annual average of any function of $\mathbf{n}$ can be expressed as a statistical expectation over the corresponding SFD $p(\mathbf{n})$.) The resulting constrained MaxEnt SFD obtained by maximising $H$ subject only to (6) is denoted by $p_{\mathrm{ME}}(\mathbf{n} \mid \bar{R})$. In general, $p_{\mathrm{ME}}(\mathbf{n} \mid \bar{R})$ is not uniform.

Can we expect $p_{\mathrm{ME}}(\mathbf{n} \mid \bar{R})$ to give realistic predictions of abundances in our plant community? After all, mean annual resource balance is only one (rather broad) ecological constraint. Abundance sequences observed in real plant communities result from complicated biological processes, and will likely only constitute a small subset of all sequences consistent with mean annual resource balance. This means that we would need to impose many additional restrictions on the possible abundance sequences before our restricted set of abundance sequences would start to resemble reality. The key point here is that we are not trying to predict the dynamical evolution of $\mathbf{n}$ from year to year, only its long-run statistics as encapsulated by sample frequencies. Many of the details governing the dynamical evolution of $\mathbf{n}$ are irrelevant for determining these sample frequencies, which will primarily depend on a smaller set of dominant ecological factors.

Here we assume that mean annual resource balance expressed in terms of the resource use of different cover types encapsulates the essential biological mechanism underlying the abundance sample frequencies in a plant community, and that other biological factors can be attributed to drift. In the next section we will calculate $p_{\mathrm{ME}}(\mathbf{n} \mid \bar{R})$ and compare some of its predictions to known ecological patterns.

\section{Results}

\subsection{Basic model properties}

The calculation of $p_{\mathrm{ME}}(\mathbf{n} \mid \bar{R})$ is straightforward using the method of Lagrange multipliers. We obtain

$p_{\mathrm{ME}}(\mathbf{n} \mid \bar{R})=Z^{-1} e^{-\beta\left(r_{1} n_{1}+\ldots+r_{C} n_{C}\right)}$

where the Lagrange multiplier $\beta$ is chosen to ensure that equation (6) holds and

$Z=\sum_{0 \leq n_{1}+\ldots+n_{C} \leq N} e^{-\beta\left(r_{1} n_{1}+\ldots+r_{C} n_{C}\right)}$

is a normalisation constant. Equation (7) implies that changing the resource availability $\bar{R}$, and thereby 
changing $\beta$, will alter the cover type abundance probabilities. When $\bar{R}$ is close to $N r_{0}$ (its smallest possible value consistent with (6)), $\beta$ will be large and positive and almost all grid cells will be occupied by the $i=0$ bare ground cover type. Increasing $\bar{R}$ decreases $\beta$ until, when $\beta=0$, all abundance vectors $\mathbf{n}$ are equally likely i.e. $p_{\mathrm{ME}}(\mathbf{n} \mid \bar{R})=p_{\mathrm{ME}}(\mathbf{n})$. Increasing $\bar{R}$ further until it is close to $N r_{C}$ (its greatest possible value consistent with (6)) will make $\beta$ large and negative and the $i=C$ cover type will dominate.

Since the joint distribution $p_{\mathrm{ME}}(\mathbf{n} \mid \bar{R})$ has a simple exponential form, if the sum over cover types could be decoupled into independent sums over the abundance of each cover type, it would be trivial to analytically evaluate $Z$, statistical moments of $p_{\mathrm{ME}}(\mathbf{n} \mid \bar{R})$ (means and variances of abundance and resource use) and the marginal distributions $p_{i}(n)$ for the abundance of each cover type $i$. However, independent summation is not possible because of equation (1). For example, the sum in equation (8) runs over abundances that satisfy $0 \leq$ $n_{1}+\ldots+n_{C} \leq N$. We have addressed this complication in two ways.

First, we have derived an alternative exact expression for $Z$ that is much easier to evaluate numerically than the sum in equation (8) (Appendix A). Whereas the number of terms in (8) grows exponentially with $C$, our alternative expression only grows quadratically with $C$. The statistical moments of $p_{\mathrm{ME}}(\mathbf{n} \mid \bar{R})$ can then be obtained by taking derivatives of $Z$ with respect to $r_{i}$ and $\beta$.

Second, we have applied the method of steepest descent (Appendix B), a standard integral approximation technique, to obtain approximate results for the case when both $N$ and $C$ are large (say $N \sim O\left(10^{3}\right)$ and $C \sim O(10)$ or greater). Our steepest descent approximation draws inspiration from Volkov et al (2004), where it was applied in a rather different ecological setting - they did not use MaxEnt and considered an upper bound on total community metabolic rate rather than a fixed average. Because the steepest descent procedure is somewhat abstract, it is more intuitive to consider the following closely related approximation. Suppose that the exact, fixed $N$ requirement (1) is replaced with a weaker constraint on the mean total grid cell abundance $N$

$$
\sum_{n_{0}, \ldots, n_{C}}^{\infty} p(\mathbf{n})\left(n_{0}+\ldots+n_{C}\right)=N .
$$

where sums over abundances now run from zero to infinity for each cover type independently. Then, the MaxEnt abundance SFD $p_{\mathrm{ME}}(\mathbf{n} \mid \bar{R})$ satisfying constraints (6) and (9) is given by

$p_{\mathrm{ME}}(\mathbf{n} \mid \bar{R}) \propto e^{-\alpha_{1} n_{1}-\ldots-\alpha_{C} n_{C}}$ where

$\alpha_{i}=\beta\left(r_{i}-\mu\right)$

and $\beta \mu$ is the Lagrange multiplier corresponding to constraint (9). Since abundances are now summed independently, the marginal distribution for the abundance of cover type $i(i=0, \ldots, C)$ is given by

$p_{i}(n)=\left(1-e^{-\alpha_{i}}\right) e^{-\alpha_{i} n}$,

and thus the mean abundances are given by

$\left\langle n_{i}\right\rangle=\frac{1}{e^{\alpha_{i}}-1}$,

while the abundance variances depend quadratically on their mean

$\sigma^{2}\left(n_{i}\right)=\left\langle n_{i}\right\rangle\left(\left\langle n_{i}\right\rangle+1\right)$.

Note that the $\left\langle n_{i}\right\rangle$ are monotonically decreasing or increasing with $i$ depending on the sign of $\beta$ : if $\beta>0$ then $\left.\left\langle n_{0}\right\rangle>\ldots\right\rangle\left\langle n_{C}\right\rangle$, and vice versa. Furthermore, note that $\mu$ changes when $\beta$ is changed in order to keep the mean total abundance $N$ fixed in equation (9); thus $\mu=\mu(\beta)$. In particular, when $\beta \rightarrow 0, \mu(\beta)$ becomes infinite at a rate that ensures $\beta \mu$ is finite and non-zero. This ensures that equations (12)-(14) are well-behaved when $\beta \rightarrow 0$.

Equations (12), (13) and (14) are identical to the results of the steepest descent approximation apart from two technical caveats:

1. While correlations between cover type abundances calculated from the relaxed constraint (9) are zero, in the steepest descent approximation these correlations are nonzero and negative,

$\sigma\left(n_{i}, n_{j}\right) \approx \frac{-\sigma^{2}\left(n_{i}\right) \sigma^{2}\left(n_{j}\right)}{\left\langle n_{0}\right\rangle^{2}+\sigma^{2}\left(n_{1}\right)+\ldots+\sigma^{2}\left(n_{C}\right)}$,

where $i, j=1, \ldots C$.

2. The steepest descent approximation does not give approximate expressions for the bare ground covariances $\sigma\left(n_{0}, n_{i}\right)$, or for the marginal probability distribution $p_{0}(n)$. The variance $\sigma^{2}\left(n_{0}\right)$ does not have the simple form given in (14). The $i=C$ cover type does satisfy (12) and (14) provided that $\beta$ is not large and negative. However, in the case $\beta \rightarrow-\infty$ (i.e. $\left.\left\langle n_{C}\right\rangle \rightarrow N\right), \sigma^{2}\left(n_{C}\right)$ will tend to zero and the marginal probability distribution $p_{C}(n)$ does not have the simple form given in (12).

In the remainder of this section, we explore some ecological implications of the predicted abundance probabilities $p_{\mathrm{ME}}(\mathbf{n} \mid \bar{R})$. Many of our results are based on the approximation (12) to (14), although these are modified when necessary according to the above caveats. 


\subsection{Species abundance distributions}

We now derive an analogue of the species abundance distribution (SAD) from our plant model. For a given group of organisms, the SAD gives the fraction of species in that group with each abundance. Empirical SADs are always peaked near the least abundant species and decrease sharply with increasing $n$, i.e. observed ecological communities always have a few abundant species and many rare ones (McGill et al, 2007). The apparent universality of this pattern has made the prediction of SADs a popular topic in theoretical ecology, and many different theories have produced realistic SADs (McGill et al, 2007). Being able to predict realistic SADs can thus be regarded as a necessary requirement of theories that predict species abundances, but a weak test of their validity (McGill et al, 2007).

Although our cover-type-based model does not predict taxonomic species abundances, which are necessary to derive SADs, it does predict cover type abundance probabilities. These probabilities can be used to calculate the analogous "SAD" $\Phi(n)$ defined as the fraction of vegetated cover types present in the community with abundance $n(n=1, \ldots, N)$. The bare ground $(i=0)$ cover type is excluded from the definition of $\Phi(n)$ because it represents the absence of plants whereas SADs are defined by the presence of organisms.

Suppose that our plant community has cover type abundances $\mathbf{n}=\left(n_{0}, \ldots, n_{C}\right)$ in a given year. Then, the number of vegetated cover types with abundance $n$ is given by

$s_{n}(\mathbf{n})=\delta_{n_{1}}^{n}+\ldots+\delta_{n_{C}}^{n}$

where $\delta_{x}^{y}$ is 1 when $x=y$ and 0 otherwise, and the total number of cover types present is

$S(\mathbf{n}) \equiv s_{1}(\mathbf{n})+\ldots+s_{C}(\mathbf{n}) \leq C$.

Here $S \leq C$ reflects the possibility that not all $C$ vegetated cover types will be present in all years.

Our model predicts cover type abundance probabilities $p_{\mathrm{ME}}(\mathbf{n} \mid \bar{R})$ rather than abundances in any given year. Thus, we define $\Phi(n)$ in terms of the expected behaviour of our plant community,

$\Phi(n)=\frac{\left\langle s_{n}\right\rangle}{\langle S\rangle} \quad n=1, \ldots, N$

where

$\left\langle s_{n}\right\rangle=p_{1}(n)+\ldots+p_{C}(n)$,

is the expected number of cover types with abundance $n$. The marginal distributions $p_{i}(n)$ are given in equation (12) (equation (12) is used for the $i=C$ cover type notwithstanding the caveats in section 3.1 , because under most conditions $\left\langle s_{n}\right\rangle$ is not sensitive to the value of $p_{C}(n)$ when $C$ is large).

The expected total number of vegetated cover types is given by

$\langle S\rangle=\left\langle s_{0}\right\rangle+\ldots+\left\langle s_{C}\right\rangle=C-\sum_{i=1}^{C} p_{i}(0)$,

while the expected total number of vegetated grid cells in the community is given by

$\left\langle N_{V}\right\rangle=N-\left\langle n_{0}\right\rangle=\langle S\rangle \sum_{n=1}^{N} n \Phi(n)$.

In terms of species abundances, $\langle S\rangle$ is analogous to species richness, and $\left\langle N_{V}\right\rangle$ is analogous to the total number of individual organisms.

Figure 1 shows a selection of predicted SADs $\Phi(n)$ plotted as rank abundance distributions, where rank is given by $\langle S\rangle(\Phi(n)+\Phi(n+1)+\ldots+\Phi(N))$ for each abundance $n$. For comparison we also plot the lognormal SAD

$\Phi_{\mathrm{LN}}(n)=a e^{-b(\ln n-c)^{2}} / n$,

where the constants $a, b$ and $c$ are fitted to an empirical SAD for tropical forest trees on Barro Colorado Island (BCI) which contains 21, 457 individual trees and 225 different species (Volkov et al, 2003). For consistency with $\Phi_{\mathrm{LN}}(n), \Phi(n)$ is calculated for $C=250$ and $N=$ 21,500 (recall $\langle S\rangle \leq C$ ). We consider the cases $\gamma=$ $\frac{1}{2}, 1,2$ to explore both $\gamma>1$ and $\gamma<1$ in equation (4).

Figure 1 shows that for all three choices of $\gamma, \bar{R}$ can be chosen such that $\Phi(n)$ is qualitatively similar to the lognormal SAD $\Phi_{\mathrm{LN}}(n)$, exhibiting a similar "S" shape. Although the species abundances expressed in $\Phi_{\mathrm{LN}}(n)$ are not strictly comparable with cover type abundances, this demonstrates that our predicted abundance patterns are broadly consistent with well-known empirical patterns of species abundance.

Interestingly, figure 1 also shows that $\Phi(n)$ changes shape depending on the resource availability $\bar{R}$. Changes in SADs along environmental gradients have been studied empirically but have received little theoretical attention (McGill et al, 2007). In Fig. 1 we show how, for $\gamma=2$, cover type abundances become more evenly distributed as $\bar{R}$ is increased from $\bar{R} / N=$ $0.005 r_{C}$ to $\bar{R} / N=0.33 r_{C}$ (which is maximally even, in the sense that $\beta=0$ and the expected abundances are equal). Since higher resource availability $\bar{R}$ will be strongly correlated with greater productivity (i.e. more biomass), this behaviour closely resembles the empirical tendency for abundances to be more even in more productive environments (McGill et al, 2007). 


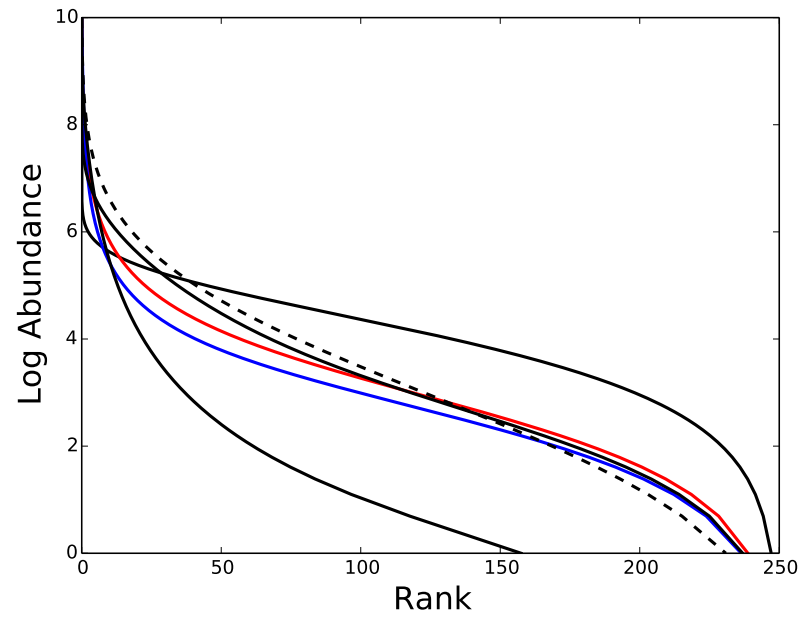

Fig. 1 Rank abundance distributions calculated from $\Phi(n)$ with $C=250$ and $N=21,500$ for: $\gamma=2$ and $\bar{R} / N=$ $0.33 r_{C}$ (upper black), $\bar{R} / N=0.05 r_{C}$ (middle black), $\bar{R} / N=$ $0.005 r_{C}$ (lower black); $\gamma=1$ and $\bar{R} / N=0.9 r_{C}$ (blue); $\gamma=\frac{1}{2}$ and $\bar{R} / N=0.92 r_{C}$ (red). The dashed line is a lognormal SAD fit to BCI data (21, 457 individual trees, 225 species) (Volkov et al, 2003).

\subsection{Stability-diversity relationships}

In this section we discuss how variances predicted by our model (which represent temporal variation) change when the number of cover types $C$ is varied. A growing number of experiments have shown that the temporal variability of community-aggregated variables, such as total biomass, abundance or resource uptake, tend to decrease with increases in species richness (Cardinale et al, 2012). Interestingly, this behaviour occurs in spite of increased variability for each species (population variability) (Tilman, 1999). Theoretical explanations for this behaviour have so far been framed in terms of three separate effects: the portfolio effect, compensatory dynamics, and overyielding (Tilman, 1999). We will discuss these three effects in turn, as well as the population variability, and relate these to the predictions of our model.

For simplicity, in this section we assume that the $i=0$ and $i=C$ cover types do not completely dominate the plant community i.e. that $\left\langle n_{0}\right\rangle / N$ and $\left\langle n_{C}\right\rangle / N$ are small compared to 1 . In this case, the expected number of vegetated cover types present in the community $\langle S\rangle$ (equation (20)) is approximately equal to $C$, and thus changing $C$ amounts to changing species richness.

It is possible to change $C$ in different ways, with different results. We consider the simplest case of changing $C$ keeping all other model parameters constant. In general this changes all resource use values $r_{i}$ according to equation (4). This can be interpreted as changing the cover type composition rather than adding/removing new cover types to an existing composition of cover types.

Our main focus will be on how changes in $C$ affect the variability of total vegetated abundance $N_{V}$, which is defined as the coefficient of variation of $N_{V}$

$V_{C}\left(N_{V}\right)=\frac{\sigma\left(N_{V}\right)}{\left\langle N_{V}\right\rangle}$

where $\sigma\left(N_{V}\right)=\sqrt{\sigma^{2}\left(N_{V}\right)}$ (the purpose of the subscript $C$ will soon be apparent). The variance of $N_{V}$ can be decomposed into two terms,

$\sigma^{2}\left(N_{V}\right)=\sum_{i=1}^{C} \sigma^{2}\left(n_{i}\right)+\sum_{i \neq j} \sigma\left(n_{i}, n_{j}\right)$,

where the first term is the summed variance of the vegetated abundances and the second is the summed covariance between them. Changes in $V_{C}\left(N_{V}\right)$ with $C$ can therefore be attributed to changes in the summed variance, the summed covariance and the expected vegetated abundance $\left\langle N_{V}\right\rangle$. These three sources of change correspond respectively to the portfolio effect, compensatory dynamics and overyielding.

\subsubsection{The portfolio effect}

The portfolio effect is a reduction in the variability of a sum of random variables (such as $V_{C}\left(N_{V}\right)$ ) as the number of those variables (i.e. $C$ ) increases, due to an averaging of their separate fluctuations. This effect is a result of the way that the summed variance $\sum_{i=1}^{C} \sigma^{2}\left(n_{i}\right)$ changes with $C$, and can thus occur in the absence of correlations between abundances or changes in $\left\langle N_{V}\right\rangle$.

Consider the following simple example (Tilman, 1999). Suppose that abundance variances obey Taylor's power law

$\sigma^{2}\left(n_{i}\right) \propto\left\langle n_{i}\right\rangle^{z}$,

that the average abundances are equal $\left\langle n_{i}\right\rangle=\left\langle N_{V}\right\rangle / C$ and that there are no abundance covariances. Then, if we change the number of cover types from $C$ to $C^{\prime}$, the new standard deviation $\sigma\left(N_{V}\right)^{\prime}$ of $N_{V}$ satisfies

$\frac{\sigma\left(N_{V}\right)^{\prime}}{\sigma\left(N_{V}\right)}=\left(\frac{C}{C^{\prime}}\right)^{(z-1) / 2}$.

Since it is generally expected that $z$ will lie between 1 and 2 in most ecosystems (Tilman, 1999), $\sigma\left(N_{V}\right)$ will generally decrease when the vegetated abundance is divided between more cover types. This decrease in $\sigma\left(N_{V}\right)$ will act to cause a decrease in $V_{C}\left(N_{V}\right)$.

The portfolio effect emerges from our model without assuming equation (25) or equal expected abundances. 
In our model, equation (25) is replaced by the predicted variances (14), which is similar to the power law (25) with a value of $z$ between 1 and 2 . Typically the expected abundances are large (of order 10 or more) because the number of different cover types will be much smaller than the number of grid cells $N / C \gg 1$, and we effectively have $\sigma^{2}\left(n_{i}\right) \approx\left\langle n_{i}\right\rangle^{2}$ (i.e. $z \approx 2$ ).

It can be shown (Appendices $\mathrm{C}$ and $\mathrm{D}$ ) that changing $C$ to $C^{\prime}$ (keeping all other parameters constant) rescales the expected abundances (which need not be equal) according to the relationship

$C^{\prime}\langle n\rangle_{r}^{\prime} \approx C\langle n\rangle_{r}$

where

$\langle n\rangle_{r}=\frac{1}{e^{\beta(r-\mu)}-1}$

is the continuous version of equation (13) (so that $\left\langle n_{i}\right\rangle=\langle n\rangle_{r_{i}}$ ) and $\langle n\rangle_{r}^{\prime}$ denotes the expected abundances when there are $C^{\prime}$ cover types. Combining (27) with (14) gives

$\frac{\sigma\left(N_{V}\right)^{\prime}}{\sigma\left(N_{V}\right)} \approx\left(\frac{C}{C^{\prime}}\right)^{1 / 2}$

similar to equation (26) when $z=2$. If the expected abundances are small (less than 1 ), then $\sigma^{2}\left(n_{i}\right) \approx\left\langle n_{i}\right\rangle$ and $V_{C^{\prime}} / V_{C} \approx 1$ so that there is no portfolio effect, similar to equation (26) when $z=1$. The ratio $\sigma\left(N_{V}\right)^{\prime} / \sigma\left(N_{V}\right)$ does not have a simple form for expected abundances intermediate between these cases $\left(1 \leq\left\langle n_{i}\right\rangle \leq 10\right)$, but since the change in variance will also be intermediate between these cases there will be a portfolio effect.

\subsubsection{Compensatory dynamics, abundance covariances}

The variance of $N_{V}$ also depends on the summed covariances $\sum_{i \neq j} \sigma\left(n_{i}, n_{j}\right)$. Covariances represent competitive (negative covariances) or mutually beneficial (positive covariances) interactions between grid cells. If the summed covariance is negative, then the overall effect of interactions between cover types is to reduce $\sigma\left(N_{V}\right)$. In this case, fluctuations in the abundances of different cover types tend to be in opposing directions i.e. abundance dynamics are "compensatory".

Although our model does not explicitly represent interactions between grid cell, they do interact implicitly through competition for space, because any increase in the abundance of one cover type must be compensated for by a decrease in the abundances of the other cover types in order to satisfy equation (1). Accordingly, the abundance covariances $\sigma\left(n_{i}, n_{j}\right)$ are all negative, as reflected in the approximate expression (15).
Figure 2 shows an example of the predicted change in the summed variances and covariances with $C$. The summed variance decreases with $C$, the summed covariance is negative but increases with $C$, and the total variance of $N_{V}$ (summed variance plus summed covariance) also decreases. This figure is remarkably similar to Fig. 2 in Tilman (1999), even though his results are obtained by entirely different methods (a resource competition model).

For comparison with Tilman (1999), the lowest values of $C$ shown in Fig. 2 are small - smaller than required for the steepest descent approximation. Thus, the exact model is solved using the alternative expression for $Z$ derived in Appendix A (see section 3.1). Figure 2 also shows the steepest descent approximation for the summed variance, which is in excellent agreement with the exact results above $C \approx 10$.

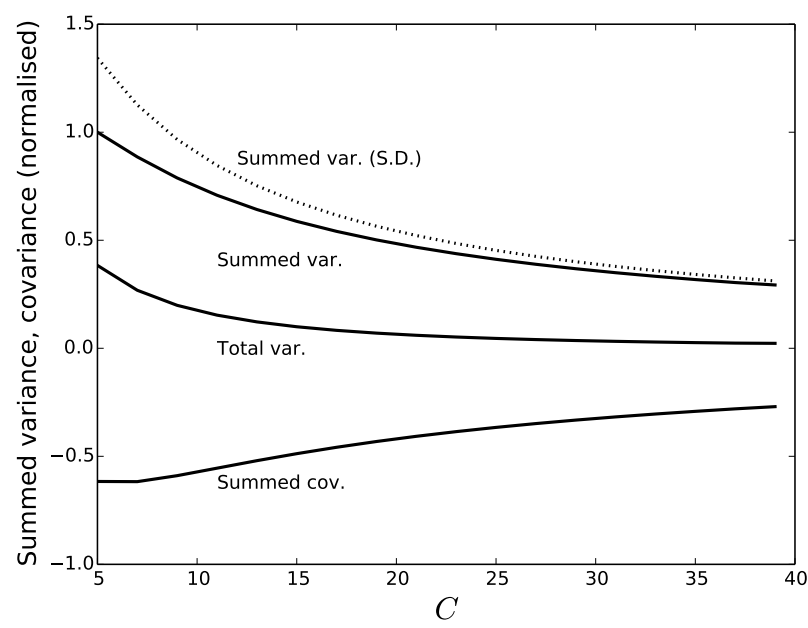

Fig. 2 Components of the variance of $N_{V}$ (eqn. (24), normalised to its maximum) versus the number of vegetated cover types $C$, obtained numerically from the exact model. Upper solid line: summed variance. Lower solid line: summed covariance. Middle solid line: total variance (summed variance + summed covariance). Dashed line: steepest descent (S.D.) approximation to summed variance from eqns. (13)(15). Here $\gamma=1, N=1000$ and $\bar{R} / N=0.4 r_{C}$.

\subsubsection{Overyielding}

Overyielding refers to an increase in overall community productivity (which can be measured in biomass or abundances) when diversity increases. Clearly, if $\left\langle N_{V}\right\rangle$ increases with $C$, this will act to reduce $V_{C}\left(N_{V}\right)=$ $\sigma\left(N_{V}\right) /\left\langle N_{V}\right\rangle$. In our model, $\left\langle N_{V}\right\rangle$ will increase with $C$ because equation (27) implies $\left\langle n_{0}\right\rangle^{\prime}=\frac{C}{C^{\prime}}\left\langle n_{0}\right\rangle$. Figure 3 shows this increase, and that $V_{C}\left(N_{V}\right)$ decreases more rapidly as a result of this overyielding. 


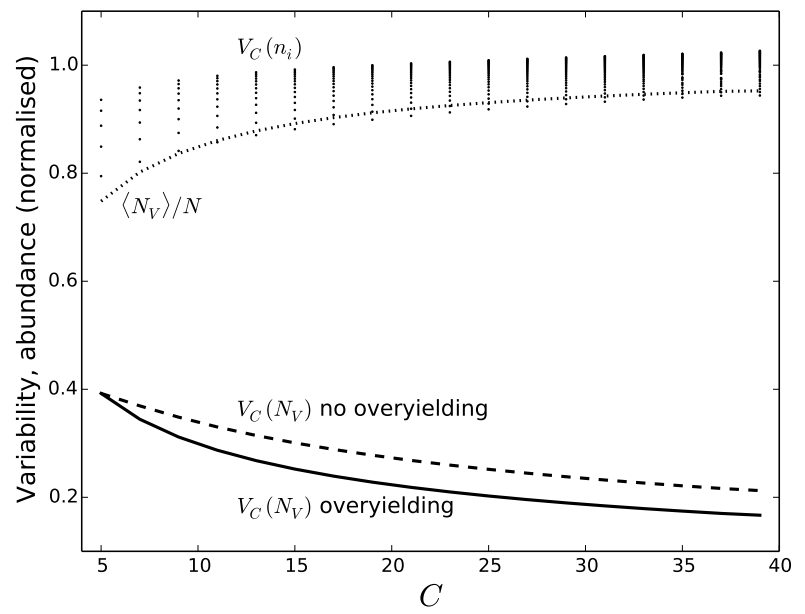

Fig. 3 Community and population variabilities versus the number of vegetated cover types $C$, obtained numerically from the exact model. Solid line: community variability $V_{C}\left(N_{V}\right)$, eqn. (23). Dashed line: $V_{C}\left(N_{V}\right)$ without overyielding (i.e. ignoring the variation in $\left\langle N_{V}\right\rangle$ and dividing by the value of $\left\langle N_{V}\right\rangle$ at $C=5$ over the whole curve). Points: population variabilities $V_{C}\left(n_{i}\right)$, eqn. (30). Dotted line: vegetated fraction $\left\langle N_{V}\right\rangle / N$. Same parameters as in fig. 2.

\subsection{Population variances}

As noted above, despite the observed tendency for total variability to decrease with increasing $C$, the variability for individual cover types (population variability)

$V_{C}\left(n_{i}\right)=\frac{\sqrt{\sigma^{2}\left(n_{i}\right)}}{\left\langle n_{i}\right\rangle}$,

tends to increase (Tilman, 1999). This fact is also consistent with the above simplified portfolio example (equations (25) and (26)) which gives

$\frac{V_{C}\left(n_{i}\right)^{\prime}}{V_{C}\left(n_{i}\right)}=\left(\frac{C}{C^{\prime}}\right)^{z / 2-1}$

in the case of no overyielding (constant $\left\langle N_{V}\right\rangle$ ). Hence, for $z<2$ the population variability increases with $C$.

Our model gives, from equation (27),

$\frac{V_{C}\left(n_{i}\right)^{\prime}}{V_{C}\left(n_{i}\right)}=\frac{\sqrt{\langle n\rangle_{r_{i}}+\frac{C^{\prime}}{C}}}{\sqrt{\langle n\rangle_{r_{i}}+1}}$,

which also increases with increasing $C$ and coincides with $(31)$ in the $z=1\left(\operatorname{small}\left\langle n_{i}\right\rangle\right)$ and $z=2\left(\right.$ large $\left.\left\langle n_{i}\right\rangle\right)$ limits. Figure 3 shows the increase in the population variability with $C$.
3.5 Phase transitions, catastrophic shifts

Decreasing the resource availability $\bar{R}$ causes the predicted bare ground fraction $\left\langle n_{0}\right\rangle / N$ to increase (because $\beta$ increases), and as $\bar{R} \rightarrow N r_{0}$ the entire community will be occupied by bare ground $\left\langle n_{0}\right\rangle / N \rightarrow 1$. When $N$ and $C$ are both large, this increase in $\left\langle n_{0}\right\rangle / N$ may occur abruptly as the resource availability $\bar{R}$ crosses a critical value. Our ecological model shares a close mathematical connection with the standard physical model of a noninteracting gas of bosons, which exhibits an analogous "phase transition"(Bose-Einstein condensation).

Figure 4 shows an example of this behaviour for two cases with $\gamma=0.5$ : a moderate- $N$ case $\left(C=10^{2}\right.$ and $N=10^{5}$, comparable to fig. 1$)$; and a high- $N$ case $\left(C=10^{5}\right.$ and $\left.N=10^{7}\right)$. It can be seen that as the normalised resource availability $\bar{R} / N r_{C}$ drops below a critical value of approximately $0.5,\left\langle n_{0}\right\rangle / N$ suddenly begins to increase from zero. Although the increase in $\left\langle n_{0}\right\rangle / N$ is abrupt in both cases, it is particularly pronounced in the high $N$ case. In the $N \rightarrow \infty$ limit, the gradient of $\left\langle n_{0}\right\rangle / N$ with respect to $\bar{R} / N r_{C}$ is discontinuous at the critical point (Appendix E).

In Appendix E we have analysed the conditions under which critical behaviour will emerge in the strict sense of the emergence of a discontinuity in the gradient of $\left\langle n_{0}\right\rangle / N$ as $N \rightarrow \infty$. This behaviour depends crucially on how $r_{1}-r_{0}$ changes with $N$. In particular, when the $r_{i}$ are given by equation (4), it can be shown that critical behaviour in this strict sense is only possible when $\gamma \leq 1$. Although ecologically realistic values of $N$ need not be large enough to approximate the $N \rightarrow \infty$ limit (e.g. the moderate $N$ case in fig 4 ), $r_{1}$ will still play a central role in determining the way that $\left\langle n_{0}\right\rangle / N$ changes with $\bar{R}$.

The abrupt change predicted in our model occurs in the rate of increase of $\left\langle n_{0}\right\rangle / N$ with $\bar{R}$. In fig. 4 , the bare ground cover fraction $\left\langle n_{0}\right\rangle / N$ increases linearly and continuously as $\bar{R} / N r_{C}$ decreases below the critical point at 0.5 towards zero. This behaviour does not represent the sudden collapse into a desert state - a sudden change in $\left\langle n_{0}\right\rangle / N$ itself - that has been documented in arid ecosystems (Scheffer et al, 2001; Kéfi et al, 2007; Scheffer et al, 2009). However, as noted above, the behaviour of $\left\langle n_{0}\right\rangle / N$ depends on the choices of the resource use values $r_{i}$, particularly $r_{1}$. It is quite possible that equation (4), which was adopted purely for the purposes of numerical illustration, does not reflect a realistic cover type composition for arid ecosystems, and that a more realistic composition would exhibit a more rapid increase in $\left\langle n_{0}\right\rangle / N$ below some critical point.

Figure 4 also shows that the critical point is associated with a peak in the variance of the number of bare 
ground grid cells $\sigma^{2}\left(n_{0}\right)$. Although increased temporal variability has been proposed as a general signature of imminent critical transitions based on bifurcation theory (Scheffer et al, 2009), few previous attempts have been made to predict this effect in ecological models. A notable exception is Volkov et al (2004), who proposed a trait-based ecological model inspired by statistical physics (but without using MaxEnt). They showed that under certain conditions the predicted variance in resource use exhibits a peak at a critical value. However, the ecological interpretation of their model is quite different from ours, and they did not attempt to explore the conditions necessary for critical behaviour.

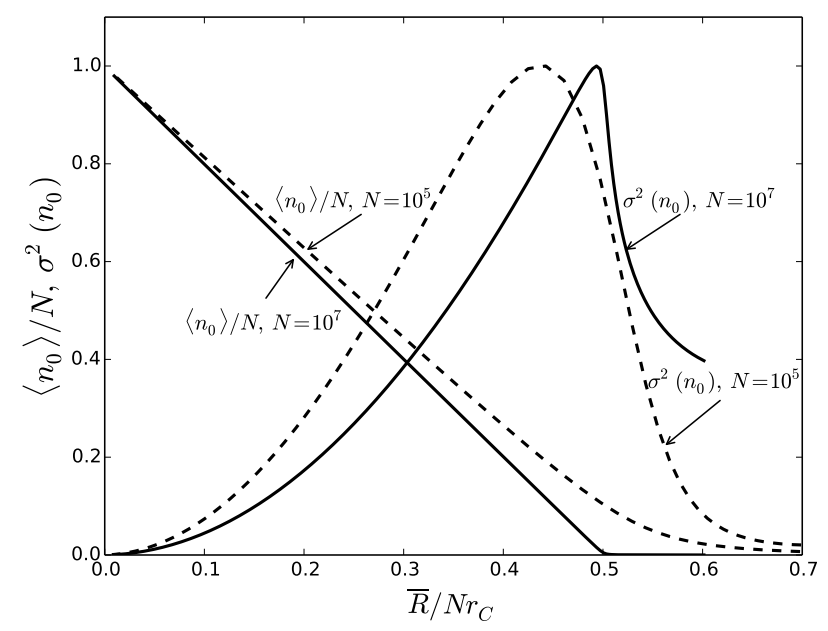

Fig. 4 Bare ground fraction $\left\langle n_{0}\right\rangle / N$ and bare ground variance $\sigma^{2}\left(n_{0}\right)$ (normalised to its maximum) versus the normalised resource availability $\bar{R} / N r_{C}$ for $\gamma=0.5$ and $C=10^{2}$ and $N=10^{5}$ (dashed); $C=10^{5}$ and $N=10^{7}$ (solid).

\section{Conclusions}

In this concluding section, we first make some further comments on the strengths and limitations of our plant community model. We then discuss some of the broader issues surrounding the application of MaxEnt and statistical mechanics in ecology, including some differences and similarities between our approach and previous studies.

Our vegetation model is based on two major idealisations: the first is that grid cell resource use covers a discrete set of values $r_{0}, \ldots, r_{C}$; the second is that the mean annual balance of this single resource encapsulates the essential biological mechanism underlying grid cell abundance probabilities, with all other processes underlying the exact dynamical evolution of grid cell abundances regarded as drift.
Treating resource use discretely may seem contrived because resource use is actually a continuous variable. However, this discreteness is simply a way to formalise the idea that grid cells can be regarded as functionally distinct if differences in their resource use are large enough. When we specify the resource use levels $r_{0}<\ldots<r_{C}$ we are effectively specifying the resource use differences that signify functional distinctness. The number of plant cover types $C$ defines the characteristic scale of those differences $\left(r_{C}-r_{0}\right) / C$. Thus, although $C$ is a free parameter in our model, it has a clear meaning as a metric of functional diversity. In contrast, a major limitation of the unified theories of biodiversity is that they require species richness as a pure numerical input i.e. without any indication of what determines species richness in the first place (McGill, 2010) (to some extent neutral theory is an exception because species richness is determined by a balance between the predicted neutral extinction rate and the assumed speciation rate). Note that in our model, the expected number of different cover types present in the community $\langle S\rangle$ is predicted, not assumed.

It is certainly possible that other factors, instead of or in addition to mean annual resource balance, are important for determining cover type abundance probabilities. Perhaps the strongest indication that mean annual resource balance alone is not sufficient to determine these probabilities is that the abundance variances predicted by our model are essentially given by Taylor's power law (25) with $z=2$, whereas it is generally expected that $z$ should lie between 1 and 2 . Values of 1.2 to 1.4 are typical in the Cedar Creek grassland experiments when looking at above-ground biomass (Tilman, 1999). These variances are a robust feature of the model; they do not depend on particular choices of $\bar{R}$ or the resource use values $r_{i}$.

However, a key strength of the model is that it predicts a wide variety of patterns with some success, including the qualitative changes in SADs along an environmental gradient, stability-diversity relationships including the opposing behaviour of community and population variability with diversity, and a critical transition in the bare ground fraction with decreasing resource availability. Furthermore, the abundance probabilities $p(\mathbf{n})$ can in principle be used to predict a wide variety of other ecological patterns. For example, the similarity in cover type composition between two local communities (e.g. abundance correlations) which have the same set of possible cover types (i.e. the same $r_{i}$ ) can in principle be obtained from the predicted $p(\mathbf{n})$ for each community. Predicting a number of different patterns in this manner is an important and often overlooked requirement of ecological models. Individual pat- 
terns in ecology are often open to multiple explanations because of generic statistical effects and the inherently limited precision and reproducibility of ecological data. Focusing on individual patterns in isolation can therefore easily lead to models with narrow scope that cannot be properly tested (and possibly rejected) - an important lesson of the theory of SADs (McGill et al, 2007).

An obvious way to improve our plant community model would be to impose additional constraints when maximising $H$ (section 2). Additional constraints may take the form of mean annual balance of other resources, similar to (6), although it is also possible that actual abundance probabilities reflect the effects of more complicated processes such as direct competition for resources or interactions between multiple trophic levels, which need not be expressible as simple average constraints over $p(\mathbf{n})$. This could potentially be an interesting avenue for future research, since constraints on MaxEnt distributions that are not simple averages are almost entirely unexplored. However, it is impossible to know a priori which biological details are essential for determining abundance probabilities (and thus which processes can be treated implicitly as drift) - this can only be determined by agreement or disagreement of the resulting predictions with observations.

As noted in section 1 , the small number of existing ecological models that have a statistical mechanics flavour differ considerably in their approaches, even within the subset of these models that use MaxEnt (notably, Kerner (1957) and Volkov et al (2004) do not use MaxEnt). Indeed, MaxEnt can be used in two conceptually distinct ways: "empirically" and "theoretically" (Dewar and Porté, 2008; McGill and Nekola, 2010). When used "empirically", MaxEnt is a data analysis method e.g. for inferring species geographic distributions from sparse presence-only data (Phillips et al, 2006). In contrast, when MaxEnt is used "theoretically", as in statistical mechanics, it becomes the statistical inference component of a model of ecosystem functioning. The empirical and theoretical uses of MaxEnt are often poorly distinguished in the literature, perhaps not surprisingly given that the mathematics of MaxEnt is the same in both cases. As a result, some ostensibly "theoretical" uses of MaxEnt can be interpreted as a sophisticated form of curve-fitting (McGill and Nekola, 2010; Bertram, 2014), much like the "empirical" applications of MaxEnt.

This problem is exacerbated by the widespread use of the information theory interpretation of MaxEnt (Pueyo et al, 2007; Harte et al, 2008; Dewar and Porté, 2008). In this interpretation, constraints represent information about some variables (e.g. information about abundances), and MaxEnt translates this information into the unique probability distribution (representing knowledge about the variables) that satisfies the imposed constraints and no others (i.e. that reflects this information alone). If the resulting probability distribution does not agree with observations, we conclude that the imposed constraints are invalid, or that other constraints are needed, or both - whatever the case, we try a different set of constraints (different "information"). Although some constraints may intuitively seem more reasonable than others, there is no definite ecological reason to prefer one set of "information" over another apart from comparing the resulting probabilities with data. This is reminiscent of a curve-fitting procedure, such as regression analysis, in which the aim is to find a mathematical relationship that captures key features of a set of observations without there needing to be any connections to the underlying processes.

In contrast, our sample frequency approach keeps the ecological significance of the MaxEnt procedure to the fore (section 2). Our constraint represents more than abstract "information" - it is a formal statement of mean annual resource balance. If we were to modify our constraints, we would be exploring the consequences of different ecological assumptions in order to try to understand the processes underlying a given set of observations, a standard theoretical practise. This distinction is not merely academic, it has definite practical repercussions. Mean annual resource balance may or may not apply in a given community, and in principle can be verified or rejected entirely independently of our model or of MaxEnt. Furthermore, using the explicit sample frequency interpretation of $p(\mathbf{n})$, new constraints could be derived starting from basic ecological postulates on the level of individual organisms, at least in principle. Constraints derived in this manner could be highly non-intuitive, and would be unlikely to emerge from guessing different averages based on what feels correct intuitively. Thus, our ecologicallybased sample frequency approach is a major distinction from the related model presented in Dewar and Porté (2008), as well as the relatively well-known model of Harte et al (2008) (another distinction is that Harte et al (2008) is formulated at a lower resolution than our model, directly predicting the proportion of species with a given abundance, rather than starting at the more detailed level of abundance probabilities $p(\mathbf{n})$ ).

\section{References}

Adler PB, HilleRisLambers J, Levine JM (2007) A niche for neutrality. Ecology Letters 10(2):95-104, DOI 10.1111/j.1461-0248.2006.00996.x 
Alexeyev V, Levich A (1997) A search for maximum species abundances in ecological communities under conditional diversity optimization. Bulletin of Mathematical Biology 59:649-677, DOI 10.1007/BF02458424

Banavar JR, Maritan A, Volkov I (2010) Applications of the principle of maximum entropy: from physics to ecology. Journal of Physics: Condensed Matter 22(6):063,101

Bartlett M (1960) Stochastic population models in ecology and epidemiology. London: Methuen and co.

Bertram J (2014) Maximum entropy models of ecosystem functioning. AIP Conference Proceedings 1636:131-136

Bertram J, Dewar RC (2013) Statistical patterns in tropical tree cover explained by the different water demand of individual trees and grasses. Ecology 94(10):2138-2144

Cardinale BJ, Duffy JE, Gonzalez A, Hooper DU, Perrings $\mathrm{C}$, Venail P, Narwani A, Mace GM, Tilman D, Wardle DA, et al (2012) Biodiversity loss and its impact on humanity. Nature 486(7401):59-67

Clark JS (2010) Individuals and the variation needed for high species diversity in forest trees. Science 327(5969):1129-1132, DOI 10.1126/science.1183506

Clark JS (2012) The coherence problem with the unified neutral theory of biodiversity. Trends in ecology and evolution 27(4):198-202

Clark JS, Dietze M, Chakraborty S, Agarwal PK, Ibanez I, LaDeau S, Wolosin M (2007) Resolving the biodiversity paradox. Ecology Letters 10(8):647-659

Dewar RC, Porté A (2008) Statistical mechanics unifies different ecological patterns. Journal of Theoretical Biology 251(3):389-403, DOI 16/j.jtbi.2007.12.007

Harte J, Zillio T, Conlisk E, Smith AB (2008) Maximum entropy and the state-variable approach to macroecology. Ecology 89(10):27002711

Hubbell SP (2001) The unified neutral theory of biodiversity and biogeography (MPB-32), vol 32. Princeton University Press

Jaynes ET, Bretthorst GL (2003) Probability theory: the logic of science. Cambridge Univ Pr

Kéfi S, Rietkerk M, Alados CL, Pueyo Y, Papanastasis VP, ElAich A, De Ruiter PC (2007) Spatial vegetation patterns and imminent desertification in mediterranean arid ecosystems. Nature 449(7159):213-217

Kerner EH (1957) A statistical mechanics of interacting biological species. The bulletin of mathematical biophysics 19(2):121-146, DOI 10.1007/BF02477883

Landau LD, Lifschitz EM (1958) Statistical Physics. Pergamon Press
Lotka AJ (1925) Elements of physical biology. Williams and Wilkins Baltimore

Lurié D, Wagensberg J (1983) On biomass diversity in ecology. Bulletin of Mathematical Biology 45(2):287 - 293, DOI http://dx.doi.org/10.1016/S00928240(83)80057-3

Maurer BA (1999) Untangling ecological complexity: the macroscopic perspective. University of Chicago Press

McGill BJ (2006) A renaissance in the study of abundance. Science 314(5800):770-772, DOI 10.1126/science. 1134920

McGill BJ (2010) Towards a unification of unified theories of biodiversity. Ecology Letters 13(5):627-642, DOI 10.1111/j.1461-0248.2010.01449.x

McGill BJ, Nekola JC (2010) Mechanisms in macroecology: Awol or purloined letter? towards a pragmatic view of mechanism. Oikos 119(4):591-603, DOI 10.1111/j.1600-0706.2009.17771.x

McGill BJ, Hadly EA, Maurer BA (2005) Community inertia of quaternary small mammal assemblages in north america. PNAS 102(46):16,701-16,706, DOI 10.1073/pnas.0504225102

McGill BJ, Etienne RS, Gray JS, Alonso D, Anderson MJ, Benecha HK, Dornelas M, Enquist BJ, Green JL, He F, Hurlbert AH, Magurran AE, Marquet PA, Maurer BA, Ostling A, Soykan CU, Ugland KI, White EP (2007) Species abundance distributions: moving beyond single prediction theories to integration within an ecological framework. Ecology Letters 10(10):995-1015, DOI 10.1111/j.14610248.2007.01094.x

Phillips SJ, Anderson RP, Schapire RE (2006) Maximum entropy modeling of species geographic distributions. Ecological Modelling 190(34):231 - 259, DOI 10.1016/j.ecolmodel.2005.03.026

Pueyo S, He F, Zillio T (2007) The maximum entropy formalism and the idiosyncratic theory of biodiversity. Ecology Letters 10(11):10171028, DOI 10.1111/j.1461-0248.2007.01096.x

Rosindell J, Hubbell SP, He F, Harmon LJ, Etienne RS (2012) The case for ecological neutral theory. Trends in Ecology and Evolution 27(4):203 - 208, DOI http://dx.doi.org/10.1016/j.tree.2012.01.004

Scheffer M, Carpenter S, Foley JA, Folke C, Walker B (2001) Catastrophic shifts in ecosystems. Nature 413(6856):591-596

Scheffer M, Bascompte J, Brock WA, Brovkin V, Carpenter SR, Dakos V, Held H, van Nes EH, Rietkerk M, Sugihara G (2009) Early-warning signals for critical transitions. Nature 461(7260):53-59

Shipley B, Vile D, Garnier (2006) From plant traits to plant communities: a statistical mechanistic ap- 
proach to biodiversity. Science 314(5800):812814

Tilman D (1999) The ecological consequences of changes in biodiversity: a search for general principles. Ecology 80(5):1455-1474

Tilman D (2004) Niche tradeoffs, neutrality, and community structure: a stochastic theory of resource competition, invasion, and community assembly. Proceedings of the National Academy of Sciences of the United States of America 101(30):10,854-10,861

Vellend M (2010) Conceptual synthesis in community ecology. The Quarterly review of biology 85(2):183206

Volkov I, Banavar JR, Hubbell SP, Maritan A (2003) Neutral theory and relative species abundance in ecology. Nature 424(6952):10351037

Volkov I, Banavar JR, Maritan A (2004) Organization of ecosystems in the vicinity of a novel phase transition. Phys Rev Lett 92:218,703, DOI 10.1103/PhysRevLett.92.218703

Zhou SR, Zhang DY (2008) A nearly neutral model of biodiversity. Ecology 89(1):248-258

\section{Appendix A: Alternative exact expression for $Z$}

In this appendix we show that the exact expression for $Z$ given by equation (8), namely

$$
\begin{aligned}
Z \equiv Z_{C} & =\sum_{0 \leq n_{1}+\ldots+n_{S} \leq N} \exp \left(-\beta\left(r_{1} n_{1} \ldots+r_{C} n_{C}\right)\right) \\
& =\sum_{n_{1}=0}^{N} \sum_{n_{2}=0}^{N-n_{1}} \ldots \sum_{n_{C}=0}^{N-n_{1}-\ldots-n_{C-1}} \exp \left(-\beta \sum_{i=1}^{C} r_{i} n_{i}\right),
\end{aligned}
$$

can be written in the numerically more convenient form

$Z_{C}=\sum_{i=1}^{C} \frac{1-x_{i}^{N+C}}{1-x_{i}} \frac{1}{\prod_{j \neq i}^{C}\left(x_{i}-x_{j}\right)}$,

where $x_{i}=\exp \left(-\beta e_{i}\right)$ and $\prod_{j \neq i}^{C}$ denotes the product over all $j=1, \ldots, C$ excluding $j=i$.

This result can be proved by induction on $C$ i.e. by showing: (i) that (34) holds when $C=1$ and (ii) that if (34) holds when there are $C-1$ cover types, then it also holds when there are $C$ cover types. Once (i) and (ii) are established, it follows that (34) applies for all C.

When $C=1,(34)$ is the standard formula for the sum of a geometric series, so (i) is true. To establish (ii), we evaluate the innermost sum in the second line of equation (33) to obtain

$$
\begin{array}{r}
Z_{C}\left(r_{1}, \ldots, r_{C}\right)=\frac{1}{1-x_{C}}\left[Z_{C-1}\left(r_{1}, \ldots, r_{C-1}\right)\right. \\
\left.-x_{C}^{N+1} Z_{C-1}\left(r_{1}-r_{C}, \ldots, r_{C-1}-r_{C}\right)\right] .
\end{array}
$$

Then, assuming that equation (34) holds for $C-1$ cover types, equation (35) implies

$Z_{C}=\sum_{i=1}^{C-1} \frac{1-x_{i}^{N+C}}{1-x_{i}} \frac{1}{\prod_{j \neq i}^{C}\left(x_{i}-x_{j}\right)}+\frac{1-x_{C}^{N+C}}{1-x_{C}} \Psi$

where

$\Psi=-\sum_{i=1}^{C-1} \frac{1}{\prod_{j \neq i}^{C}\left(x_{i}-x_{j}\right)}$.

Equation (36) must agree with equation (34) for $C$ cover types in order for (ii) to hold. Thus, we need to show that $\Psi=1 / \prod_{j \neq C}^{C}\left(x_{C}-x_{j}\right)=1 / \prod_{j=1}^{C-1}\left(x_{C}-x_{j}\right)$, or equivalently, that the function

$$
\begin{aligned}
f\left(x_{C}\right) & =\Psi\left(x_{C}\right) \prod_{j=1}^{C-1}\left(x_{C}-x_{j}\right) \\
& =\sum_{i=1}^{C-1} \prod_{j \neq i}^{C-1} \frac{\left(x_{C}-x_{j}\right)}{\left(x_{i}-x_{j}\right)}
\end{aligned}
$$

is equal to unity. Since the product $\prod_{j \neq i}^{C-1}$ has $C-2$ terms, $f\left(x_{C}\right)$ is a polynomial in $x_{C}$ of order at most $C-$ 2. By inspection, $f\left(x_{C}\right)=1$ when $x_{C}=x_{1}, \ldots, x_{C-1}$. Therefore, the polynomial $f\left(x_{C}\right)-1$ (which has the same order as $\left.f\left(x_{C}\right)\right)$ has at least $C-1$ roots. However, a non-zero polynomial of order $n$ can have at most $n$ roots. It follows that $f\left(x_{C}\right)-1$ is identically zero i.e. that $f\left(x_{C}\right)$ is a constant with value 1 . This completes the proof of equation (34).

\section{Appendix B: Steepest descent approximation}

Here we apply the method of steepest descent to derive a simpler approximate expression for $Z_{C}$. The accuracy of this approximation improves if $N$ is made larger, becoming exact in the limit $N \rightarrow \infty$. Our technique is adapted from Volkov et al (2004).

We start by writing the constrained sum $\sum_{0 \leq n_{1}+\ldots+n_{C} \leq N}$ in the form $\sum_{n_{1}, \ldots, n_{C}}^{\infty} \Theta\left(N-\sum_{i=1}^{C} n_{i}\right)$ where $\Theta$ is the Heaviside step function and the sums over $n_{1}, \ldots, n_{C}$ now go independently from zero to infinity. Using the integral representation $\Theta(x)=\frac{1}{2 \pi i} \int_{\gamma} e^{z x} / z d z$ we have

$$
\begin{aligned}
Z_{C} & =\sum_{n_{1}, \ldots, n_{C}}^{\infty} \exp \left(-\beta \sum_{i=1}^{C} r_{i} n_{i}\right) \Theta\left(N-\sum_{i=1}^{C} n_{i}\right) \\
& =\frac{1}{2 \pi i} \int_{\gamma} e^{N z-\ln z} \prod_{i=1}^{C} \sum_{n=0}^{\infty} e^{\left(-\beta r_{i}-z\right) n} d z \\
& =\frac{1}{2 \pi i} \int_{\gamma} e^{g(z)} d z
\end{aligned}
$$


where

$g(z)=N z-\ln z-\sum_{i=1}^{C} h\left(\beta r_{i}+z\right)$,

$h\left(\beta r_{i}+z\right)=-\ln \sum_{n=0}^{\infty} e^{-\left(\beta r_{i}+z\right) n}$,

and the contour of integration $\gamma$ is any vertical line in the complex plane intersecting the real axis at a positive value.

Expanding $g(z)$ to second order around the saddle point $z_{0}$ lying on the (positive) real axis defined by the condition $g^{\prime}\left(z_{0}\right)=0$ yields

$Z_{C} \approx \frac{e^{g\left(z_{0}\right)}}{\sqrt{2 \pi g^{\prime \prime}\left(z_{0}\right)}}$,

where $g^{\prime}\left(z_{0}\right)=0$ implies

$N=\frac{1}{z_{0}}+\sum_{i=1}^{C} h_{i}^{\prime}$

with $h_{i}=h\left(\alpha_{i}\right)$ and $\alpha_{i}=\beta e_{i}+z_{0}$.

The accuracy of the steepest descent approximation relies on $g$ being sharply peaked at $z_{0}$ along the steepest descent contour i.e. $\left|g^{\prime \prime}\left(z_{0}\right)\right| \gg 1$. This condition is satisfied when $N$ is very large. To see this, note that

$g^{\prime \prime}\left(z_{0}\right)=\frac{1}{z_{0}^{2}}-\sum_{i=1}^{C} h_{i}^{\prime \prime}$,

where

$h_{i}^{\prime}=\frac{1}{e^{\alpha_{i}}-1} \quad h_{i}^{\prime \prime}=-h_{i}^{\prime}\left(h_{i}^{\prime}+1\right)$.

Using equation (44), it can be shown that $g^{\prime \prime}\left(z_{0}\right)>N-$ $1 / 4$ by minimising $g^{\prime \prime}\left(z_{0}\right)$ with respect to the variables $x_{0}=1 / z_{0}>0$ and $x_{i}=h_{i}^{\prime}>0(i=1, \ldots, C)$ where $x_{0}+\sum_{i=1}^{C} x_{i}=N$ (ignoring the functional dependence between them). Thus, for a given $N$ the minimum in $g^{\prime \prime}\left(z_{0}\right)$ with respect to $\beta$ (when the steepest descent approximation is worst) is bounded below by $N$. For the remainder of this Appendix, $N$ is assumed to be large.

\section{Abundance means and variances}

Equation (42) can be used to obtain simple expressions for many statistical moments of interest provided (as will be shown) that $C$ is large, say $C \sim O(10)$ or greater (assumed for the remainder of this Appendix).
The expected $n_{i}$ are given by

$$
\begin{aligned}
\left\langle n_{i}\right\rangle & =-\left.\frac{1}{\beta} \frac{\partial \ln Z_{C}}{\partial e_{i}}\right|_{N, \beta} \\
& \approx h_{i}^{\prime}+\left.\frac{1}{2 \beta} \frac{\partial \ln g^{\prime \prime}\left(z_{0}\right)}{\partial e_{i}}\right|_{N, \beta} \quad \quad i=1, \ldots, C \\
& =h_{i}^{\prime}+\frac{g^{\prime \prime \prime} h_{i}^{\prime \prime}+g^{\prime \prime} h_{i}^{\prime \prime \prime}}{2 g^{\prime \prime 2}}, \quad
\end{aligned}
$$

where the derivative is taken holding $N$ and $\beta$ fixed and $h_{i}^{\prime \prime \prime}=-h_{i}^{\prime \prime}\left(2 h_{i}^{\prime}+1\right)$.

The second term in (46) is obtained by taking the derivative of equation (43) to obtain

$\left.\frac{\partial z_{0}}{\partial e_{i}}\right|_{N, \beta}=\beta h_{i}^{\prime \prime} / g^{\prime \prime}\left(z_{0}\right)$.

This term is at most proportional to $h_{i}^{\prime} / C$ (if $N$ is approximately evenly partitioned) or $h_{i}^{\prime} / N$ (if $1 / z_{0}$ is of order $N$ ). Consequently, the mean cover type abundances are given by

$\left\langle n_{i}\right\rangle \approx h_{i}^{\prime}, \quad i=1, \ldots, C$

and thus, from equation (43),

$\left\langle n_{0}\right\rangle \approx 1 / z_{0}$

The variance of $n_{i}$ is given by

$$
\begin{aligned}
\sigma^{2}\left(n_{i}\right) & =-\left.\frac{1}{\beta} \frac{\partial\left\langle n_{i}\right\rangle}{\partial e_{i}}\right|_{N, \beta} \\
& \approx-h_{i}^{\prime \prime}-\frac{h_{i}^{\prime \prime 2}}{g^{\prime \prime}} .
\end{aligned}
$$

Again, the second term vanishes if $N$ and $C$ are large for $i=1, \ldots, C$ provided that $\beta$ is not large and negative, giving

$\sigma^{2}\left(n_{i}\right) \approx-h_{i}^{\prime \prime} \quad i=1, \ldots, C$

However, as $\beta \rightarrow-\infty$ and the $i=C$ cover type starts to dominate the community $\left(h_{C}^{\prime} \approx\left\langle n_{C}\right\rangle \rightarrow N\right)$, we have $-h_{C}^{\prime \prime 2} / g^{\prime \prime} \rightarrow h_{C}^{\prime \prime}$. In this case

$\sigma^{2}\left(n_{C}\right)=-h_{C}^{\prime \prime}-\frac{h_{C}^{\prime \prime 2}}{g^{\prime \prime}} \quad$ if $\quad \beta \rightarrow-\infty$

Note that $\sigma^{2}\left(n_{C}\right) \rightarrow 0$ as $\beta \rightarrow-\infty$; this is because the total grid cell abundance $N$ is fixed and so the variance in $n_{C}$ must vanish in the limit that all grid cells have cover type $C$.

The covariance between $n_{i}$ and $n_{j}$ is given by

$$
\begin{aligned}
\sigma\left(n_{i}, n_{j}\right) & =-\left.\frac{1}{\beta} \frac{\partial\left\langle n_{i}\right\rangle}{\partial e_{j}}\right|_{N, \beta} \\
& \approx-\frac{h_{i}^{\prime \prime} h_{j}^{\prime \prime}}{g^{\prime \prime}} .
\end{aligned}
$$


Marginal abundance distributions

The marginal abundance distribution for cover type $i$ is defined by

$p_{i}(n)=\sum_{n_{i}=n} p_{\mathrm{ME}}(\mathbf{n} \mid \bar{R})$,

where the sum is taken over all $\mathbf{n}$ with $n_{i}=n$. Assuming that $\beta$ is not large and negative and evaluating higher derivatives of $\ln Z_{C}$, we obtain, as in equations (48) and (51)

$\left.\frac{\partial^{m} \ln Z_{C}}{\partial\left(-\beta r_{i}\right)^{m}}\right|_{N} \approx \frac{\partial^{m} h_{i}}{\partial \alpha_{i}^{m}} \quad i=1, \ldots, C$,

because $h_{i}^{\prime \prime 2} / g^{\prime \prime}$ can be neglected at each stage as before. Equation (55) defines the cumulants of $p_{i}(n)$, which can be used construct a series representation of $p_{i}(n)$ (an Edgeworth series). Thus, we can approximate $p_{i}(n)$ with a probability distribution that has the same cumulants. Consequently, we have

$p_{i}(n) \approx\left(1-e^{-\alpha_{i}}\right) e^{-\alpha_{i} n} \quad i=1, \ldots, C$

\section{Total resource use}

The expected total resource use is given by

$$
\begin{aligned}
\langle r\rangle & =-\left.\frac{\partial \ln Z_{C}}{\partial \beta}\right|_{N} \\
& =\left\langle\sum_{i=1}^{C} r_{i} n_{i}\right\rangle=\sum_{i=1}^{C} r_{i}\left\langle n_{i}\right\rangle \\
& \approx \sum_{i=1}^{C} r_{i} h_{i}^{\prime}
\end{aligned}
$$

and its variance by

$$
\begin{aligned}
\sigma^{2}(r) & =-\left.\frac{\partial\langle r\rangle}{\partial \beta}\right|_{N} \\
& =\sigma^{2}\left(\sum_{i=1}^{C} r_{i} n_{i}\right)=\sum_{i=1}^{C} r_{i}^{2} \sigma^{2}\left(n_{i}\right)+\sum_{i \neq j} r_{i} r_{j} \sigma\left(n_{i}, n_{j}\right) \\
& \approx-\sum_{i=1}^{C} r_{i}^{2} h_{i}^{\prime \prime}-\frac{\left(\sum_{i=1}^{C} r_{i} h_{i}^{\prime \prime}\right)^{2}}{g^{\prime \prime}}
\end{aligned}
$$

\section{Appendix C: Continuous resource spectrum ap- proximation}

When the overall density of resource use levels $C / r_{C}$ is large, we may approximate sums over species label $i$ with integrals over $r$ as follows. We assume that the resource use spectrum is "smooth" in the sense that it can be written in the form

$r_{i}=r_{C} g(i / C)$

where $g$ is any continuous, differentiable and monotonically increasing function with $g(0)=0$ and $g(1)=1$. Then the the sum of any function of resource use $f\left(r_{i}\right)$ can be approximated as an integral

$\sum_{i} f\left(r_{i}\right) \approx \int_{r_{0}}^{r_{C}} f(r) \rho(r) d r$

where the density of states $\rho(r)$ (the density of resource use levels in the vicinity of $r$ ) is defined by

$\rho(r)=\frac{C}{r_{C} g^{\prime}\left(g^{-1}\left(r / r_{C}\right)\right)}$,

where $g^{-1}$ is the inverse of $g$. For example, in equation (4) $g(i / C)=(i / C)^{\gamma}$ and the corresponding density of states is $\rho(r)=C\left(r / r_{C}\right)^{\frac{1}{\gamma}-1} / \gamma r_{C}$.

Some care needs to be taken handling the endpoints of the resource use spectrum (i.e. $r=r_{0}$ and $\left.r=r_{C}\right)$. When considering sums involving cover type abundances, say $f\left(r_{i}\right)=\left\langle n_{i}\right\rangle$, the first or last terms in the sum on the left of equation (60) may be large relative to the other terms $\left(\beta \gg 0\right.$ for large $\left\langle n_{0}\right\rangle$, $\beta \ll 0$ for a large $\left.\left\langle n_{C}\right\rangle\right)$. This means that the function $f(r)=1 /\left(e^{\beta(r-\mu)}-1\right)$ will be sharply peaked at either of the endpoints. In this case equation (60) may not be valid for ecologically realistic values of $C$

Furthermore, if $\rho$ vanishes at either endpoint (because $g^{\prime}(x)$ diverges to infinity), then these potentially large endpoint terms will not contribute to the integral on the right of (60) and this approximation will not be valid no matter how large $C / r_{C}$. In these cases the endpoint terms must be handled separately from the integral. For example, if $\left\langle n_{0}\right\rangle$ is large compared to the other expected abundances and $g(i / S)=(i / C)^{\alpha}$ where $0<\alpha<1$, then $\rho(0)=0$ and we have

$\sum_{i=0}^{C}\left\langle n_{i}\right\rangle \approx\left\langle n_{0}\right\rangle+\int_{r_{0}}^{r_{C}} 1 /\left(e^{\beta(r-\mu)}-1\right) \rho(r) d r$

provided that $C / e_{C}$ is sufficiently large.

We define a normalised density of states $\bar{\rho}$ by

$\bar{\rho} \equiv \rho / C$

where $\bar{\rho}(r)$ integrates to unity (to a good approximation i.e. neglecting terms of order $1 / C)$. 


\section{Appendix D: Rescaling of abundances with changes in $C$}

Here we prove equation (27) assuming that $C$ is large enough that sums over resource use can be approximated by integrals (Appendix C), that $r_{0}, r_{C}, \bar{R}$ and $\bar{\rho}$ remain fixed when $C$ is changed, and that the proportion of grid cells occupied by the $i=0$ and $i=C$ cover types $\left\langle n_{0}\right\rangle / N$ and $\left\langle n_{C}\right\rangle / N$ are small.

We define a continuous expected abundance function by $\langle n\rangle_{r}=1 /\left(e^{\beta(r-\mu)}-1\right)$. This function is monotonically decreasing for $\beta>0$, constant when $\beta=0$, and monotonically increasing for $\beta<0$. When the number of cover types in the community is changed from $C$ to $C^{\prime}, \beta$ and $\mu$ will also change and, since $\bar{R}$ is fixed, the resulting abundance function $\langle n\rangle_{r}^{\prime}$ satisfies

$C \int_{r_{0}}^{r_{C}} r\langle n\rangle_{r} \bar{\rho} d r \approx \bar{R} \approx C^{\prime} \int_{r_{0}}^{r_{C}} r\langle n\rangle_{r}^{\prime} \bar{\rho} d r$.

By assumption, $\left\langle n_{0}\right\rangle / N$ and $\left\langle n_{C}\right\rangle / N$ are small, which implies that $\langle n\rangle_{r}$ and $\langle n\rangle_{r}^{\prime}$ contribute significantly to the integrals in (64) over the entire resource use domain rather than being sharply peaked near the endpoints $r_{0}$ or $r_{C}$. Consequently, changing $C$ cannot induce a relative redistribution of the expected abundances $\langle n\rangle_{r}$ between resource levels as this would bias the integrand on the right hand side of (64) in one direction, violating the constancy of $\bar{R}$. This implies

$C^{\prime}\langle n\rangle_{r}^{\prime} \approx C\langle n\rangle_{r}$

Note that if $\langle n\rangle_{r}$ was sharply peaked at its endpoints $r_{0}$ or $r_{C}$, then the integrals in (64) will be dominated by the behaviour of $\langle n\rangle_{r}$ and $\langle n\rangle_{r}^{\prime}$ near those endpoints. In this case the relationship between functions the $\langle n\rangle_{r}$ and $\langle n\rangle_{r}^{\prime}$ will be more complicated than the simple ratio (65).

\section{Appendix E: Condition for criticality}

There is a close mathematical parallel between our model and the canonical model of an ideal Bose gas. Replacing resource use values $r_{i}$ with energy levels, equations (12)-(14) are identical to the corresponding expressions for the number of bosons occupying different energy levels. This suggests that our model may exhibit behaviour analogous to the Bose-Einstein Condensate (BEC) phase transition. The main difference between our model and an ideal Bose gas in terms of its phase transitions is that the resource use "spectrum" $r_{i}$ (e.g. equation (4)) may be different from the energy spectrum of bosons - the dependence of $r_{i}$ on $i$ could be different, and there is a largest resource use $r_{C}$ (energy spectra are usually treated as unbounded). Here we determine the requirements on the $r_{i}$ for critical behaviour in our model.

The total number of grid cells can be divided into bare ground and vegetated components

$N=N_{0}(\beta, \mu)+N_{V}(\beta, \mu)$,

where, from equation (43), we have $N_{0}=\left\langle n_{0}\right\rangle=-1 / \beta \mu$ and $N_{V}=\sum_{i=1}^{C}\left\langle n_{i}\right\rangle$ (to simplify notation we drop the expectation brackets on $\left.\left\langle N_{V}\right\rangle\right)$. We will restrict our attention to $\beta>0$ because we are primarily interested in the transition of the community from a primarily vegetated state to domination by bare ground. The following arguments will hold for $\beta<0$ with minor modifications, although in this case the phase transition reflects domination by the $i=C$ cover type rather than bare ground.

Figure 5 shows an example of $N_{0}(\beta, \mu)$ and $N_{V}(\beta, \mu)$ as functions of $\mu$ for two fixed values of $\beta, \beta=1$ and $\beta=2.5$, with $\gamma=2 / 3$ in equation (4) and $N=C=1000$. It can be seen that $N_{0} / N=-1 / N \beta \mu$ is essentially zero everywhere because $N \beta \gg 1$, except for in the close vicinity of $\mu=0$ where it increases sharply. For each value of $\beta$, only one value of $\mu$ is consistent with equation (66). This defines a relationship $\mu(\beta)$ for a given $N$. In the case $\beta=1, \mu(\beta=1)$ is not close to zero and $N_{V} \approx N$ while $N_{0} / N \approx 0$. However, when $\beta=2.5, N_{V}$ is smaller than $N$ for all $\mu, \mu(\beta=2.5) \approx 0$ and $N_{0} / N$ is not small. Between these two cases there exists a critical case $\beta=\beta_{c} \approx 1.96$ where both $N_{V} \approx N$ and $\mu\left(\beta_{c}\right) \approx 0$. If $\beta$ is made larger than $\beta_{c}$, the contribution from $N_{0}$ in (66) becomes significant. Because the $1 / N \beta \mu$ singularity is so sharp, the onset of this increase in $N_{0} / N$ from zero is very sudden. Thus, $N_{0} / N$ will increase suddenly when the mean annual resource availability $\bar{R}$ (which determines $\beta$ ) drops below a critical value.

More generally, we can define $\beta_{c}$ as the solution to the equation

$N_{V}\left(\beta_{c}, 0\right)=N$.

The value of $N_{V}$ at $\beta_{c}$ can be written as

$N_{V}\left(\beta_{c}, \mu_{c}\right) \equiv(1-\epsilon) N$

where $\mu_{c}=\mu\left(\beta_{c}\right)$ and $\epsilon=N_{0}\left(\beta_{c}, \mu_{c}\right) / N$. In the above example, the critical behaviour near $\beta_{c} \approx 1.96$ was a result of $\epsilon$ being small (i.e. $N_{V}\left(\beta_{c}, \mu_{c}\right) \approx N$ ) due to $N$ being large. More generally, $\beta_{c}$ defined by $(67)$ is a critical point if and only if it is possible to make $\epsilon$ tend to zero in the limit $N \rightarrow \infty$. The change in $N_{0}$ at a critical point $\beta_{c}$ becomes so sudden as $\epsilon \rightarrow 0$ that $\partial N_{0} / \partial \beta$ will not be continuous at $\beta_{c}$. 


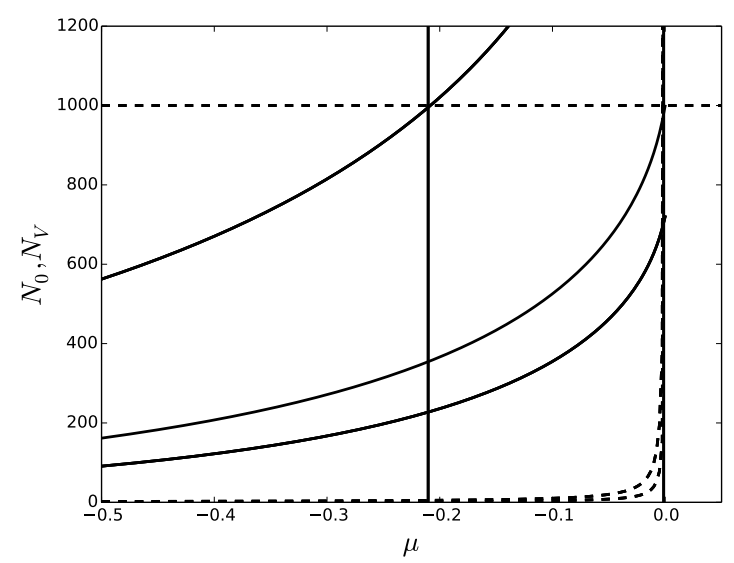

Fig. 5 Solid curves: $N_{V}(1, \mu)$ (upper), the critical case $N_{V}\left(\beta_{c} \approx 1.96, \mu\right)$ (middle) and $N_{V}(2.5, \mu)$ (lower). Dashed curves: $N_{0}(1, \mu) / N$ (upper) and $N_{0}(2.5, \mu) / N$ (lower). Horizontal dashed line: $N=1000$. Vertical lines: $\mu(\beta=1)$ (left) and $\mu(\beta=2.5)$ (right). Here $r_{i}=\left(\frac{i}{C}\right)^{2 / 3}$ and $N=C=1000$.

For a given $\beta_{c}$ defined by equation (67), whether $\beta_{c}$ is a critical point or not (i.e. whether it is possible to make $\epsilon$ tend to zero) depends on how the lowest vegetated resource use $r_{1}$ varies with $N$ when taking the $N \rightarrow \infty$ limit (if at all). Specifically, $\beta=\beta_{c}$ is a critical point if and only if

$\lim _{N \rightarrow \infty} r_{1} N=\infty$

where the limit is taken with $\beta_{c}$ held fixed. We prove this result below.

First, we show that $\lim _{N \rightarrow \infty} r_{1} N=\infty$ implies $\epsilon \rightarrow$ 0 . If $\lim _{N \rightarrow \infty} r_{1} N=\infty$, then $\lim _{N \rightarrow \infty} r_{1} N \epsilon$ is either finite or infinite. In the finite case, $\epsilon \rightarrow 0$. In the infinite case, from the definition of $\epsilon$, the ratio

$-\frac{r_{1}}{\mu_{c}}=\beta_{c} r_{1} N \epsilon$

is also infinite (recall $\beta_{c}>0$ by assumption). It follows that that $N_{V}\left(\beta_{c}, \mu_{c}\right) \rightarrow N_{V}\left(\beta_{c}, 0\right)=N$, i.e. $\epsilon \rightarrow 0$, because $\left\langle n_{1}\right\rangle \approx 1 /\left(e^{\beta_{c} r_{1}}-1\right)$ and similarly for $\left\langle n_{2}\right\rangle, \ldots,\left\langle n_{C}\right\rangle$.

Conversely, $\lim _{N \rightarrow \infty} \epsilon=0$ implies $\lim _{N \rightarrow \infty} r_{1} N=$ $\infty$. Suppose that $\lim _{N \rightarrow \infty} r_{1} N$ is not infinite. Then $\lim _{N \rightarrow \infty} r_{1} N \epsilon=0$. From equation (70) this implies that $r_{1}$ becomes much smaller than $-\mu_{c}$ as $N$ increases, so that $\left\langle n_{1}\right\rangle \approx 1 /\left(e^{-\beta_{c} \mu_{c}}-1\right)$. Thus the value of $\left\langle n_{1}\right\rangle$ will be quite different from its value if $\mu$ is set to zero, i.e. $1 /\left(e^{\beta_{c} r_{1}}-1\right)$. Since $\beta_{c}>0,\left\langle n_{1}\right\rangle$ makes the largest contribution to $N_{V}\left(\beta_{c}, \mu_{c}\right)$, which means that $N_{V}\left(\beta_{c}, \mu_{c}\right)$ will be not be equal to $N_{V}\left(\beta_{c}, 0\right)=0$. Thus, $\lim _{N \rightarrow \infty} \epsilon=0$ is only possible if $\lim _{N \rightarrow \infty} r_{1} N=\infty$. This completes our proof.
Let us consider the implications of (69) in the case of the power law resource use spectrum (4). If $C / r_{C}$ is large, we have the integral approximation (Appendix B)

$N=N_{V}\left(\beta_{c}, 0\right) \approx \frac{C}{\gamma\left(\beta_{c} r_{C}\right)^{1 / \gamma}} \int_{\beta_{c} r_{1}}^{\beta_{c} r_{C}} \frac{y^{1 / \gamma-1}}{e^{y}-1} d y$,

where $r_{1}=r_{C} / C^{\gamma}$. Equation (71) implies that to keep $\beta_{c}$ fixed, $N$ and $C$ are related by an increasing function $N=N(C)$ (assuming fixed $r_{C}$ ). By evaluating the behaviour of the integral (71) near $r=r_{1}$, we obtain

$r_{1} N \sim \begin{cases}C^{1-\gamma} & \text { if } \gamma<1 \\ \ln (C) & \text { if } \gamma=1 \\ \text { const. } & \text { if } \gamma>1\end{cases}$

Therefore, a postive $\beta_{c}$ defined by equation (67) can only be a critical point when $\gamma \leq 1$.

In ecological terms, increasing $N$ could represent dividing the area of the community into smaller grid cells or increasing the total community area. In the former case, the lowest per-grid cell vegetated resource use will simply be divided by $N$, so $r_{1} N$ would remain constant. Thus, critical behaviour is not simply an artefact of choosing a fine grid resolution. However, $r_{1}$ may decrease as the total community area increases because more cover types may be possible in a larger area. In the simplest case, $r_{1}$ would just remain constant and $r_{1} N$ would increase with $N$. 